\title{
STEREO IMAGE ANALYSIS USING CONNECTED OPERATORS
}

\author{
Albert Oliveras, Philippe Salembier and Luis Garrido \\ Universitat Politècnica de Catalunya, Campus Nord - D5 \\ C/ Gran Capità s/n, 08034-BARCELONA, Spain \\ albert@gps.tsc.upc.es
}

\begin{abstract}
Connected operators are increasingly used in image processing due to their properties of simplifying the image with various criteria, without loosing contour's information. These properties are related to the connected operator approach that either preserves or completely eliminates a determined connected component, according to an established criterion of analysis. In this paper we will define a new connected operator for stereo images. The goal is to simplify one of the images (left) in the sense that the operator will eliminate the image components that are not present at a determined location in the other image (right). This filter let us select in a stereo image, objects as a function of their distance from the observer (for instance used in auto guided vehicles).
\end{abstract}

\section{CONNECTED OPERATORS}

We start by a brief introduction on connected operators and then present the original contributions of the paper: Matching criterion definition, "spatial tolerance" parameter, non-increasingness of the operator and its efficient implementation.

Connected operators for a gray level image can be created by using binary connected operators in combination with thresholding and stacking operations [1]. A binary connected operator implies two steps: first, the analysis of each connected component of the binary image and second, the decision on the elimination or the preservation of each connected component.

For some operators the analysis step relies on a measurement on each binary connected component [2][3]. An alternative approach makes measurement on the gray level image over the zone defined by a connected component. This approach defines a connected operator too because the operator interacts with the gray level image by means of flat zones [4][5] (preserve or eliminate connected components). The main difference is

This work was supported in part by the CICYT of the Spanish government: TIC95-1022-C05 how they get the measure associated to a connected component. Two representative examples of the two different approaches are the area opening [6] and the volumic operator [7] respectively.

\section{MATCHING CONNECTED OPERATOR FOR STEREO IMAGES}

One of the methods used in stereo vision to get the depth information of the objects, is based on the four following steps:

- Take two images separated by a baseline

- Identify similar points between the two images

- Use the inverse perspective transform to derive the two lines on which the world point lies

- Intersect the lines

The hardest part of this method is the second step, where we have to find the portions of the first image (left) which best match in the second image (right) and assign a measure of "disparity" between the two regions of the images. Generally, the disparity measure of the image parts relies on templates of fixed geometry (rectangular or square). In the context of connected operators the measure of disparity will be based on the connected components.

The objective of the connected operator in stereo image analysis is to eliminate the image components from one image (left) that do not match with a similar component in the other image (right). This operator will eliminate the bright objects that do not makes a perfect match, by duality, applying the operator to the complemented image and complement the result, we obtain the operator that interacts with the dark objects.

In order to measure the "disparity" a projection model should be introduced to deal with the geometrical corrections of the reference image. For example the position of all objects at a given distance from the observer is modified between the left and right images by the same type of geometrical transformation (a translation if the two cameras are parallel on the same baseline). This model can vary from simple translation for all positions of the image, to a specific 


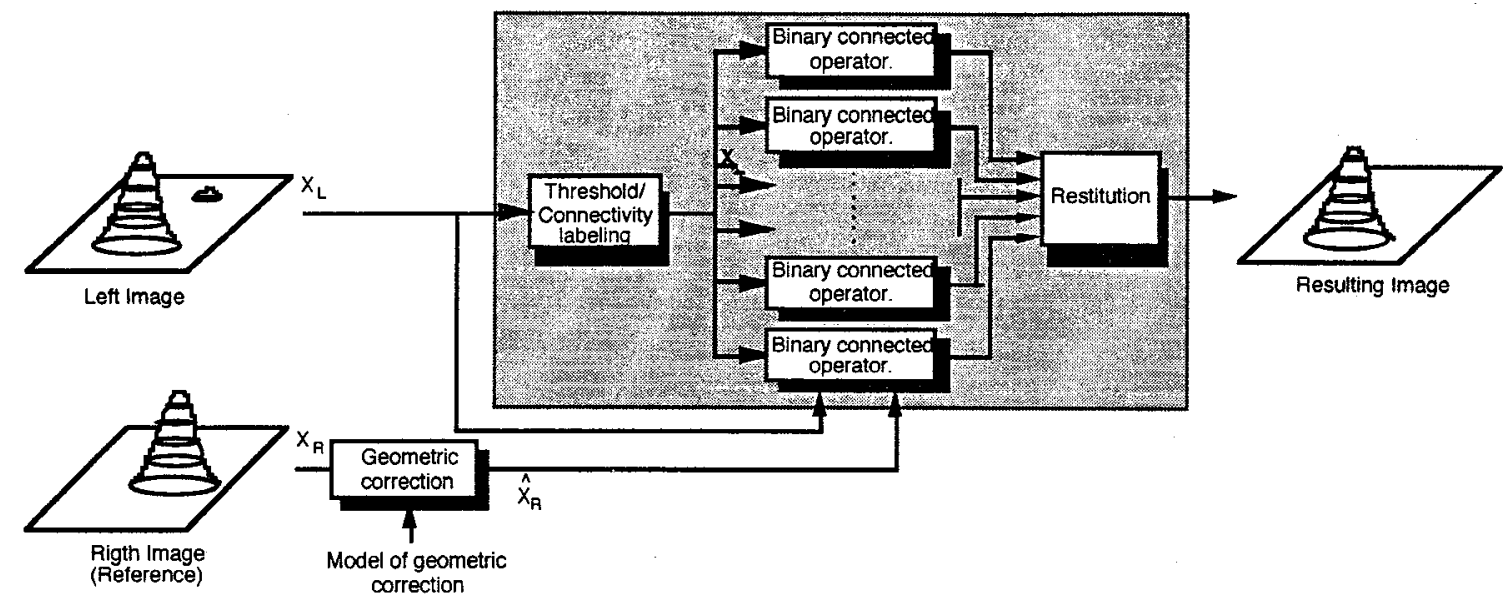

Figure 1: Blocs diagram for the Stereo image matching filter.

translation for each position of the image $\left(\Delta_{i}, \Delta_{j}\right)$. Formally, the matching criterion is function of the image to be filtered $X_{L}$ (left image) an the geometrical correction of the reference image (right image) $X_{R}\left(i-\Delta_{i}, j-\Delta_{j}\right)$. The first step of the gray level connected operator decomposes the original image into a set of binary images by thresholding for all gray level values $\lambda$ (p.e. $\lambda=[0.255]$ for 8 -bits quatized images). The measure of matching is evaluated for each connected component $C C_{x}$, and it is only defined for binary images. A connected component $C C_{x}$ is the region of a binary image that all its points satisfies the connectivity definition and contains the point $x$ (or marker $x$ ). Then the matching criterion for a connected component $C C_{x}\left(X_{L, \lambda}\right)$ of the binarized image $X_{L, \lambda}$ by thresholding of $X_{L}$ at level $\lambda$, is given by:

$$
M_{L, R}\left(C C_{x}\left(X_{L, \lambda}\right)\right)=\frac{\sum_{i, j \in C C_{x} \mid}\left|X_{L \lambda}(i, j)-X_{R}\left(i-\Delta_{i}, j-\Delta_{j}\right)\right|}{\sum_{i, j \in C C_{x}}\left(X_{L \lambda}\right)}
$$

The processing of an image (left) is performed as follows (figure 1): the image is decomposed by thresholding in a set of binary images $X_{L, \lambda}$ and each connected component labeled. To determine if a connected component $C C_{x}\left(X_{L, \lambda}\right)$ should be preserved or not, the mean gray level difference defined by equation (1), $M_{L, R}\left(C C_{x}\left(X_{L, \lambda}\right)\right)$ is computed between two images over the spatial domain defined by the connected component under consideration $\left(C C_{x}\left(X_{L, \lambda}\right)\right)$, as shown in figure 2.

The components that do not make a perfect match are eliminated and the other components preserved. In some applications a degree of "spatial tolerance" should be considered to preserve an object making a good matching without being at the exact location or having a different gray level value due to its reflectance. This notion of spatial tolerance is introduced in the matching criterion modifying the difference $\left|X_{L}-X_{R}\right|$ as follows:

$$
\left|X_{L}-X_{R}\right|= \begin{cases}X_{L}-\delta\left(X_{R}\right) & \text { if } X_{L}>\delta\left(X_{R}\right) \\ 0 & \text { if } \varepsilon\left(X_{R}\right) \leq X_{L} \leq \delta\left(X_{R}\right) \\ \varepsilon\left(X_{R}\right)-X_{L} & \text { if } X_{L}<\varepsilon\left(X_{R}\right)\end{cases}
$$

where $\varepsilon$ and $\delta$ denote respectively the erosion and the dilation operations.

The size of the structuring element used in this dilation and erosion, define the degree of "spatial tolerance" that is permitted as a perfect matching.

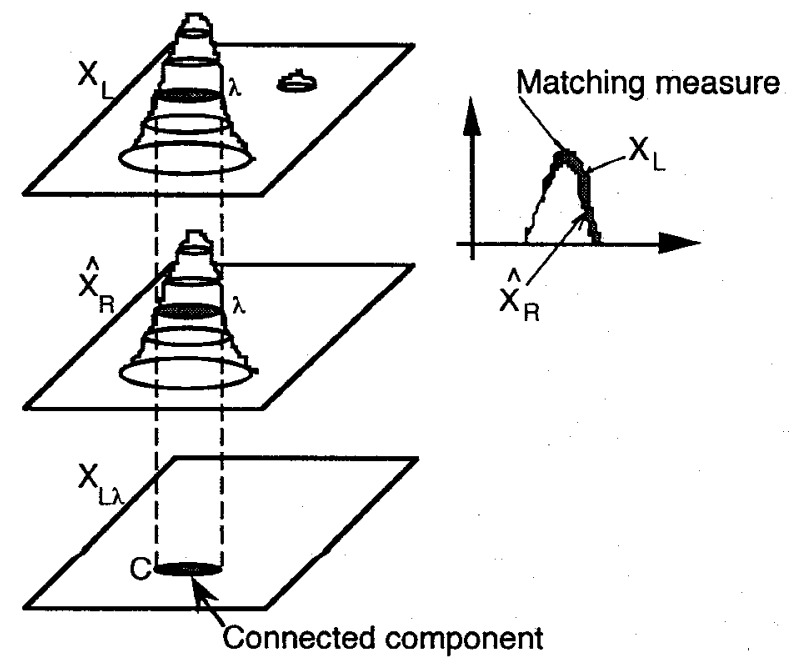

Figure 2: Detail of how to compute the matching measure over a connected region with volumic representation of images.

Note that the matching criterion is not increasing. This leads to a non-coherent decision to preserve or not, some of the connected components at different levels $\lambda$ 


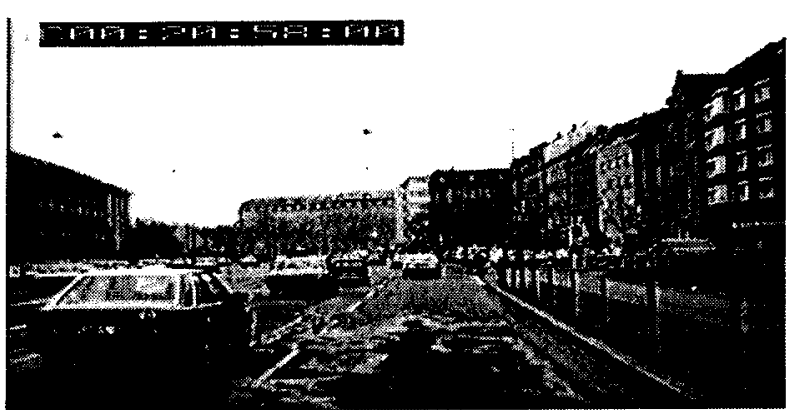

a) Original Left Image

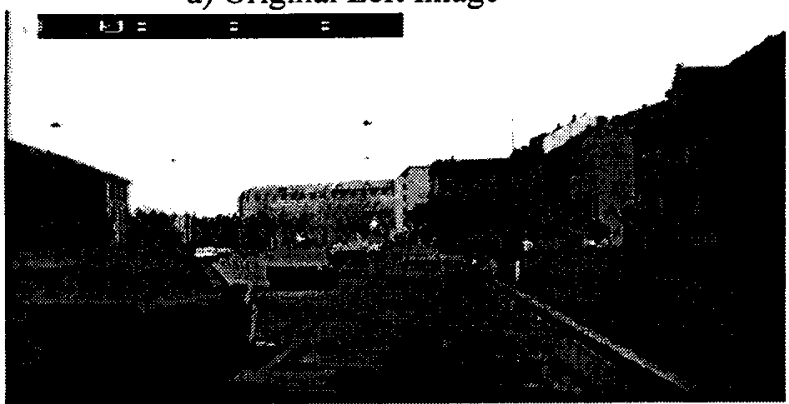

c) Result of the matching operator

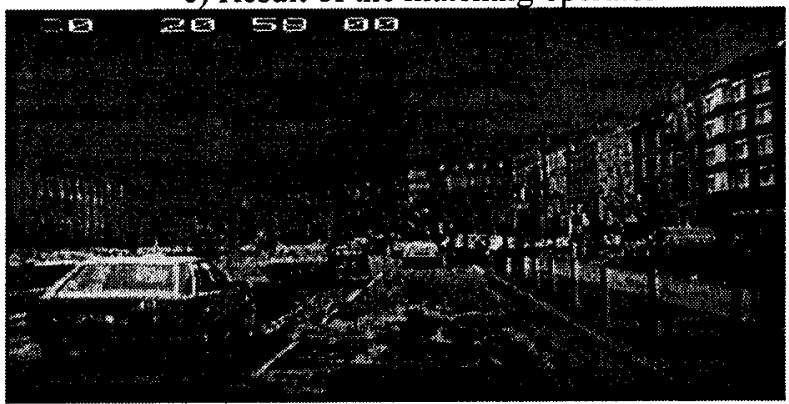

e) Left Residue

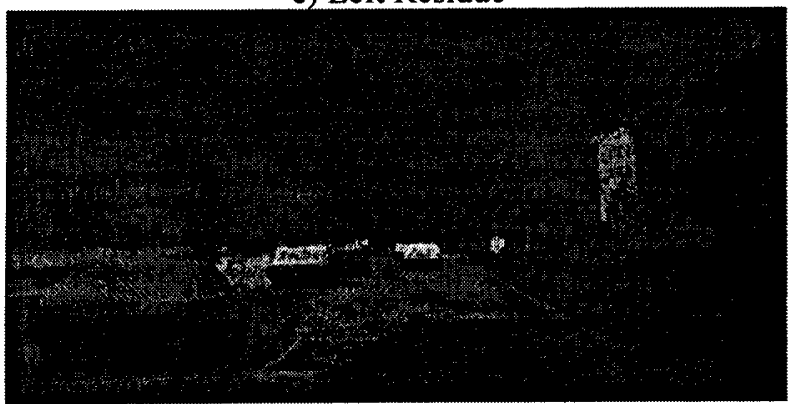

h) Result of the matching operator on residue $3 \mathrm{e}$

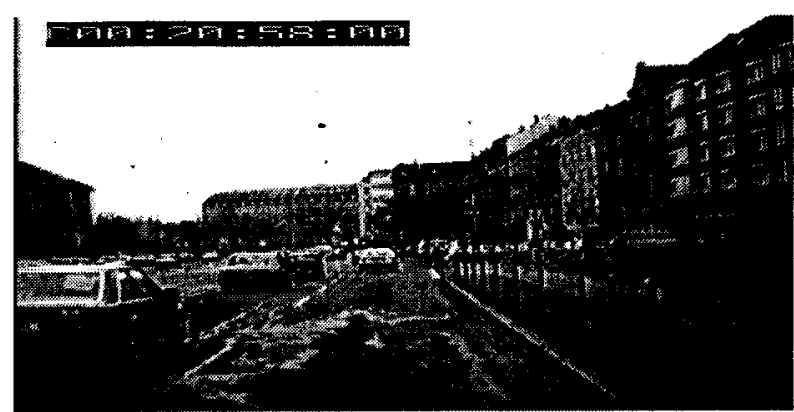

b) Original Right Image

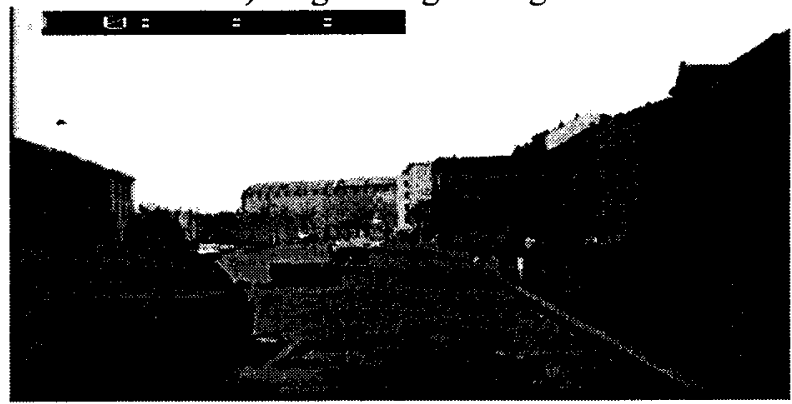

d) Result of the matching operator and its dual

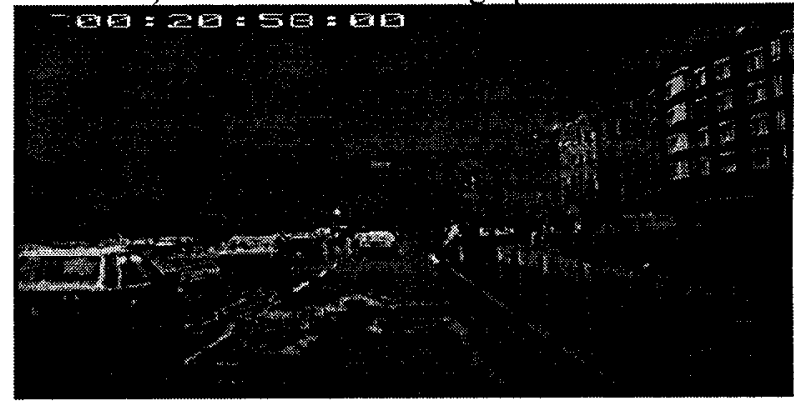

f) Right residue

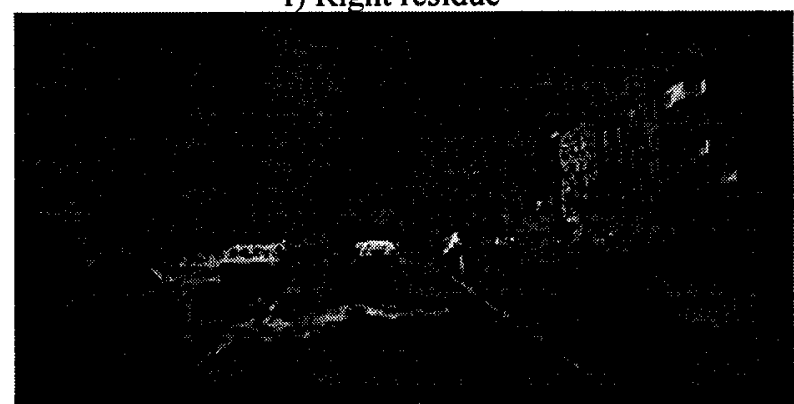

i) Result of the matching operator on residue $3 \mathrm{f}$

Figure 3: Example of depth oriented decomposition

associated to an object. The problem of converting this non-coherent decision into a coherent decision can be solved as a dynamic programming problem [8]. This solution improves the robustness of the operator.

A time efficient implementation of this type of connected filters can be obtained through a Max-Tree image representation [8].

\section{RESULTS}

In figure 3.a and 3.b we show the original images. Figure 3.c gives an example of the output of the operator, where a translation $(\Delta x=30, \Delta y=3)$ has been applied on the right images "geometrical correction", and a "spatial tolerance" of size 3 pixels is permitted . In this case, bright objects that are close to the observer have been removed. Starting from this result, dark objects can be 
eliminated by the dual operator (applying the operator to the complemented image and complement the result), as shown in fig. 3.d.

The difference between the result of the operator and its dual applied to the original image pairs is shown in $3 \mathrm{e}$. A similar result can be obtained for the left image, and is presented in figure $3 \mathrm{f}$. In these images, called residue, one can see how the objects that are close to the observer are preserved. Note in particular how the distant objects as the sky or the background buildings have been removed.

Applying the depth operator with the appropriate "geometrical correction", a translation of $(\Delta x=32, \Delta y=3)$ to the residue images 3 .d and $3 . \mathrm{f}$ we can extract the objects that are in the medium depth range (fig $3 . \mathrm{h}, \mathrm{i}$ ).

\section{CONCLUSIONS}

In this paper a new connected operator for depth information processing in stereo images is presented. This operator is an extension to stereo image processing of the connected operators for still images. A new matching criterion for a connected component and its spatial tolerance has been defined and applied to the depth estimation problem in stereo images. The examples illustrate the possibilities of the operator to detect the objects at a different depth position.

\section{REFERENCES}

[1] A. Oliveras, P. Salembier "Generalized Connected Operators" Visual Communications and Image Processing, VCIP'96, volume 2727, pages 761-773, Orlando (FL), USA, March 1996.

[2] E. Breen, R. Jones, "An attribute-based approach to mathematical morphology" In International Symposium on Mathematical Morphology, (Invited Paper), pages 41-48, Atlanta (GA), USA, May 1996.

[3] P. Salembier, A. Oliveras. "Practical extensions of connected operators". In International Symposium on Mathematical Morphology, (Invited Paper), pages 98-110, Atlanta (GA), USA, May 1996.

[4] J. Serra, P. Salembier. "Connected operators and pyramids". In SPIE, editor, Image Algebra and Mathematical Morphology, volume 2030, pages 6576, San Diego (CA), USA, July 1993.

[5] P. Salembier, J. Serra." Flat zones filtering, connected operators and filters by reconstruction". IEEE Transactions on Image Processing, 3(8):11531160 , August 1995.

[6] L. Vincent. "Gray scale area opening and closings, their efficient implementation and applications". First Workshop on Mathematical Morphology and its Applications to Signal Processing, pages 22-27, Barcelona, Spain May 1993.
[7] C. Vachier. "Extraction de caracteristiques, segmentation d'image et morphologie matematique". $\mathrm{Ph}$. Thesis, Ecole superieure des mines de Paris, France. Dec 1995.

[8] L. Garrido, A. Oliveras, P. Salembier "Motion analysis of image sequences using connected operators" VCIP-97, San Jose (CA) USA, February 1997. 